\title{
Co-delivery of doxorubicin and siRNA for glioma therapy by a brain targeting system: angiopep-2 modified poly (lactic-co-glycolic acid) nanoparticles
}

Lei Wang ${ }^{1}$, Yongwei Hao ${ }^{1}$, Haixia Li, Yalin Zhao, Dehui Meng, Dong Li, Jinjin Shi, Hongling Zhang, Zhenzhong Zhang* and Yun Zhang*

School of Pharmaceutical Sciences, Zhengzhou University, 100 Kexue Avenue, Zhengzhou, 450001, PR China.

1. Lei Wang and Yongwei Hao contributed equally to this work.

*Correspondent: Prof. Zhenzhong Zhang and Prof. Yun Zhang, School of Pharmaceutical Sciences, Zhengzhou University, NO.100 Kexue Avenue, Zhengzhou, Henan Province 450001, PR China. Tel: 86-371-67781910; Fax: 86-371-67781908;

Email: zhangzz08@126.com; zhang_yun@ymail.com 
Table 1 Characterization of nanoparticles ( $\mathrm{n}=3)$.

\begin{tabular}{llll}
\hline Preparations & \multicolumn{1}{c}{ Mean size } & Polydispersity index (PDI) & Zeta potential (mv) \\
\hline NANG/PLGA/DOX & 169.5 & 0.18 & $42.0 \pm 2.2$ \\
NANG/PLGA/DOX/siRNA & 170.8 & 0.22 & $37.0 \pm 2.2$ \\
ANG/PLGA/DOX & 185.0 & 0.25 & $38.6 \pm 4.4$ \\
ANG/PLGA/DOX/siRNA & 190.5 & 0.19 & $30.2 \pm 3.6$ \\
\hline
\end{tabular}

Note: Results presented are mean \pm SD $(n=3)$.

(1)

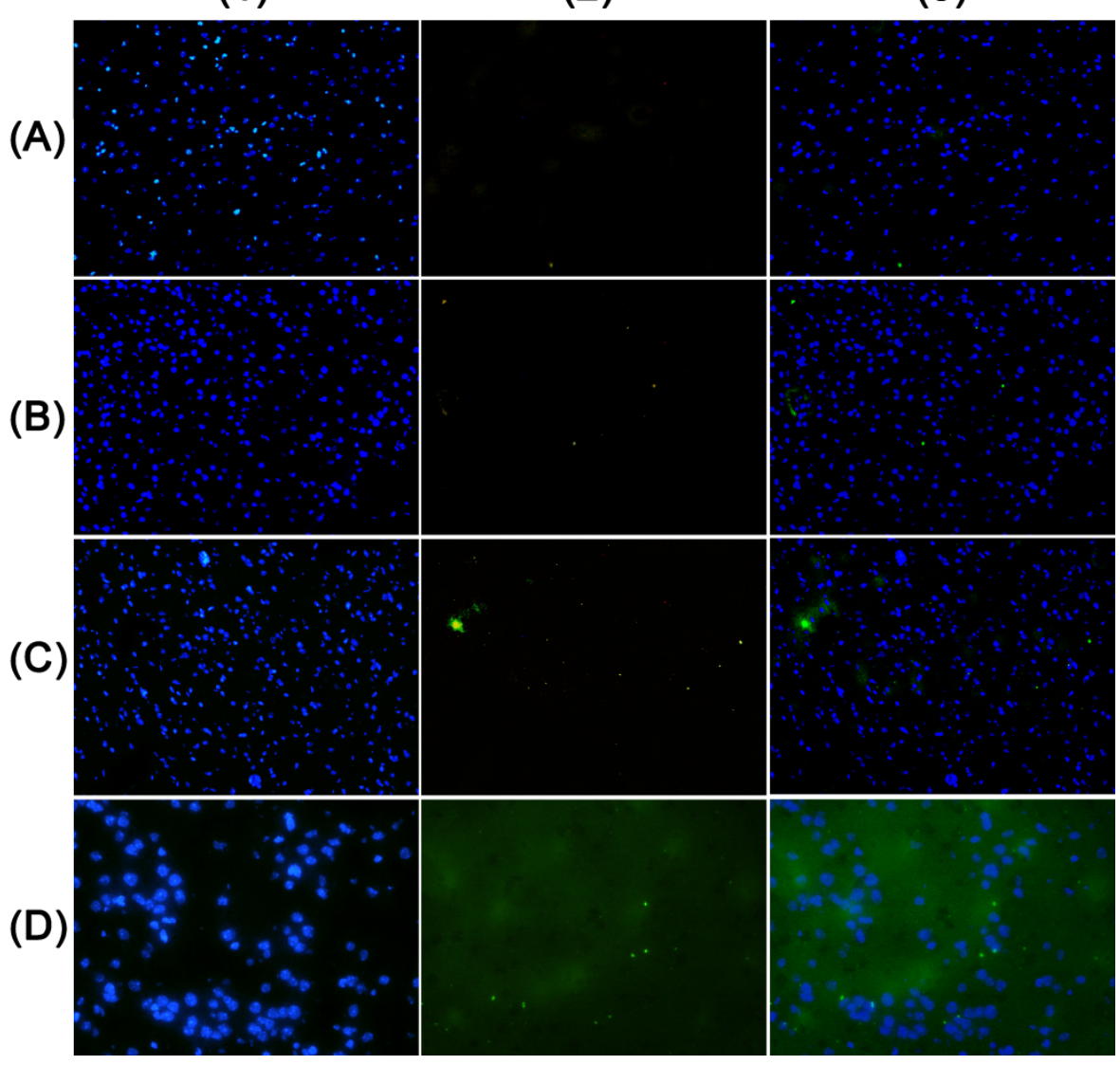

Fig. 1. Representative fluorescence microscopic images of the brain tissues from mice treated intravenously with non-targeting NPs labelled with coumarin-6 (NANG/PLGA/Coumarin-6 NPs/siRNA). (A) 1 h $(200 \times)$; (B) 2 h $(200 \times)$; (C) 3h (200 $\times$ ); (D) 3h (400×). Column (1): The nuclei in blue fluorescence are stained with DAPI. Column (2): Fluorescein isothiocyanate channels showing the green fluorescence from coumarin-6-loaded PLGA NPs distributed in brain tissues. Column (3): Merged channel of fluorescein isothiocyanate and DAPI. 
(1)

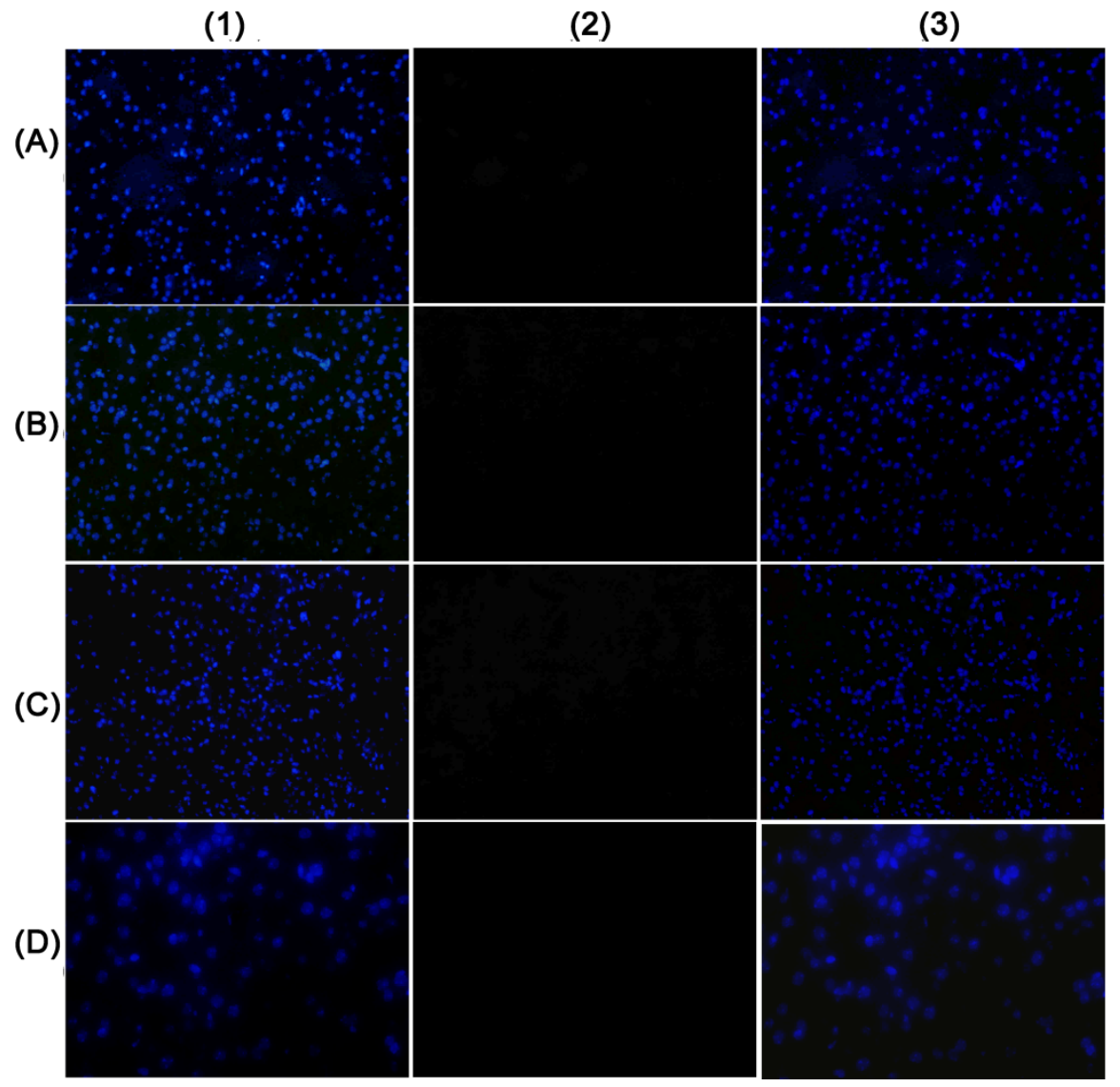

Fig. 2. Representative fluorescence microscopic images of the brain tissues from mice treated intravenously with coumarin-6 solution. (A) 1 h (200 ×); (B) 2 h (200 ×); (C) 3 h $(200 \times)$; (D) $3 \mathrm{~h}(400 \times)$. Column (1): The nuclei in blue fluorescence are stained with DAPI. Column (2): Fluorescein isothiocyanate channels showing the green fluorescence from coumarin-6-loaded PLGA NPs distributed in brain tissues. Column (3): Merged channel of fluorescein isothiocyanate and DAPI. 